\title{
Search Overview
}

Search:

Date/Time done:

Database(s):

Restriction Info:

Filters:

Percentage Completed:

Number of Hits: search6

Sat Mar 07 10:08:52 2020

CSD version 5.41 (November 2019)

No refcode restrictions applied

None

$100 \%$

16

Summary of queries used. Search found structures that:

match
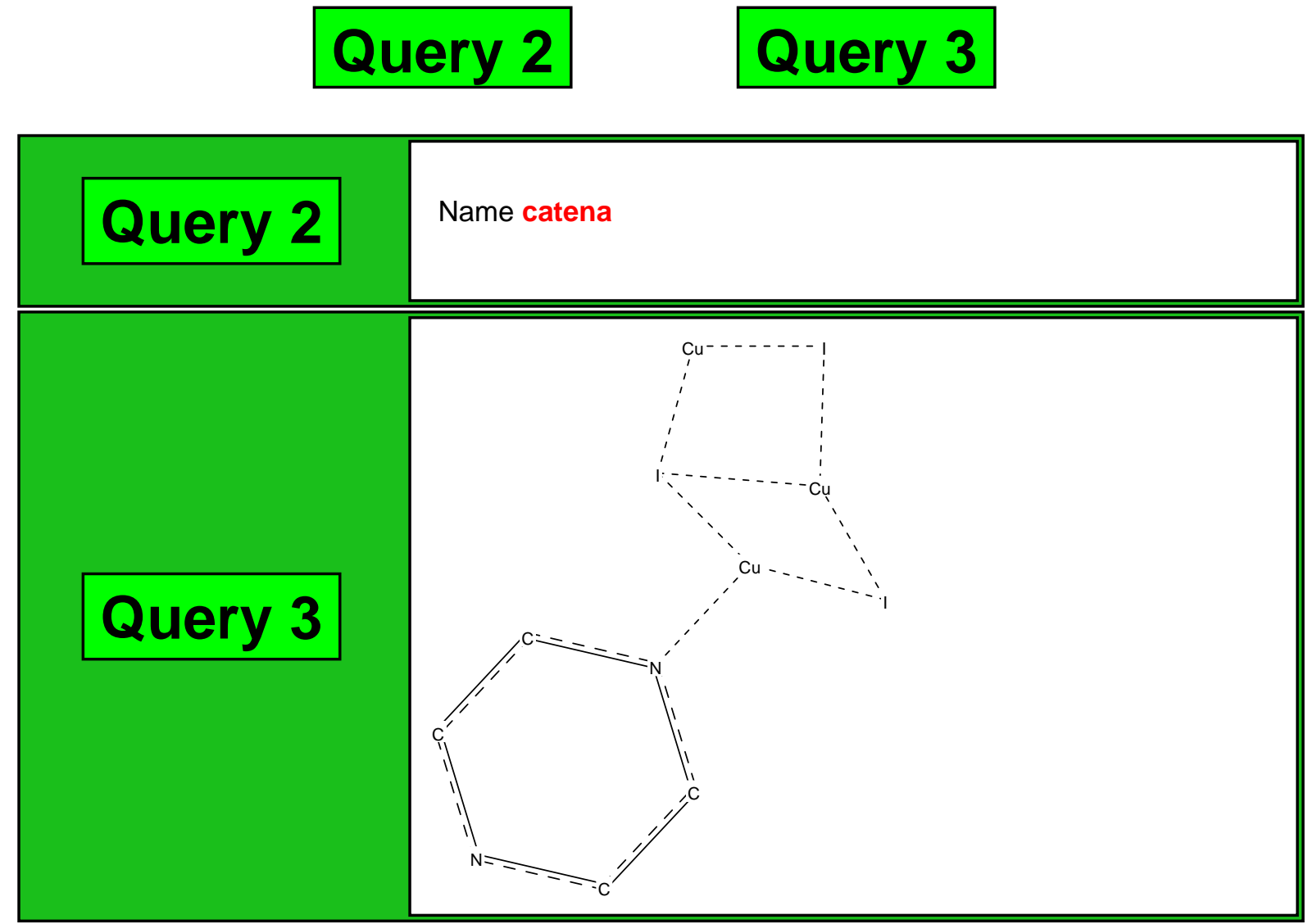


\section{Search: search6 (Sat Mar 07 10:08:52 2020): Hits 1-4}

AGIYEU01

\begin{tabular}{|c|c|c|c|c|c|c|}
\hline Reference: & \multicolumn{6}{|c|}{$\begin{array}{l}\text { A.J.Blake, N.R.Brooks, N.R.Champness, P.A.Cooke, } \\
\text { M.Crew, A.M.Deveson, L.R.Hanton, P.Hubberstey, D.Fenske, } \\
\text { M.Schroder (1999) Crystal Engineering ,2,181 }\end{array}$} \\
\hline Formula: & \multicolumn{6}{|c|}{$\left(\mathrm{C}_{4} \mathrm{H}_{4} \mathrm{Cu}_{2} \mathrm{I}_{2} \mathrm{~N}_{2}\right) \mathrm{n}$} \\
\hline Compound Name: & \multicolumn{6}{|c|}{ catena-(bis $\left(\mu_{3}\right.$-iodo)-( $\mu_{2}$-pyrazine-N,N')-di-copper(i)) } \\
\hline $\begin{array}{l}\text { Space Group: } \\
\text { Space Group No.: }\end{array}$ & $\begin{array}{l}P-1 \\
2\end{array}$ & $\begin{array}{l}\text { Cell: } \\
\left(\dot{A},{ }^{\circ}\right)\end{array}$ & $\begin{array}{ll}a & 4.134(0) \\
\alpha & 109.72(3)\end{array}$ & $\begin{array}{lr}\boldsymbol{b} & 7.058(1) \\
\beta & 101.31(3)\end{array}$ & & $\begin{array}{l}8.041(1) \\
96.54(3)\end{array}$ \\
\hline R-Factor (\%): & 4.82 & Temp & $\operatorname{rre}(K): 203$ & Density $(\mathrm{g} / \mathrm{cm}$ & & 3.604 \\
\hline
\end{tabular}

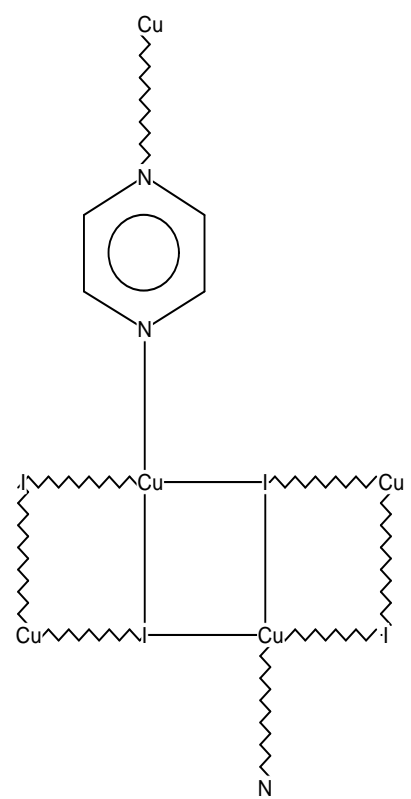

Parameters
Fragment 1
DIST1 (D)

BASBAA

$\begin{array}{ll}\text { Reference: } & \text { Jin-Cheng Wu, Liang Zhao, De-Xian Wang, } \\ \text { Mei-Xiang Wang (2012) Inorg.Chem. ,51,3860 }\end{array}$

Formula:

$$
\left(\mathrm{C}_{40} \mathrm{H}_{40} \mathrm{Cu}_{5} \mathrm{I}_{5} \mathrm{~N}_{24}\right) \mathrm{n}
$$

Compound Name: $\quad$ catena-(bis $\left(\mu_{4}-2,4,6,8\right.$-tetramethyl-2,4,6,8-tetraza-1,3,5,7(2,6)tetrapyrazinacyclooctaphane)-bis $\left(\mu_{3}\right.$-iodo)-tris $\left(\mu_{2}\right.$-iodo)-penta-copper unknown solvate)

Synonym: $\quad$ catena-(bis $\left(\mu_{4}\right.$-tetramethylazacalix[4]pyrazine)-bis $\left(\mu_{3}\right.$-iodo)-tris $\left(\mu_{2}\right.$ iodo)-penta-copper unknown solvate)

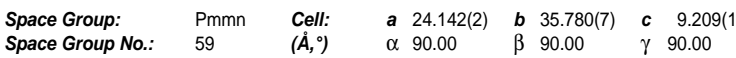

R-Factor (\%): $\quad 7.29 \quad$ Temperature(K): $113 \quad$ Density $\left(\mathrm{g} / \mathrm{cm}^{3}\right): 1.511$

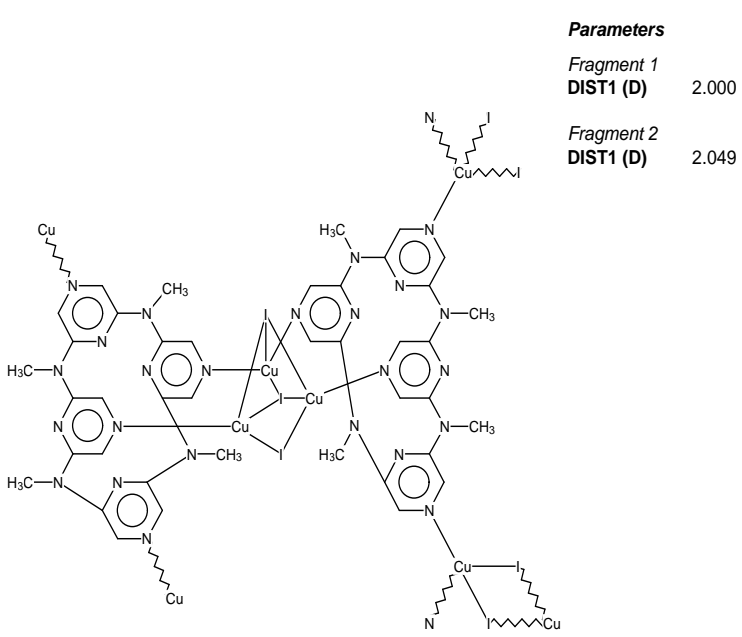

AGIYEU02

\begin{tabular}{|c|c|c|c|c|c|c|}
\hline Reference: & \multicolumn{6}{|c|}{$\begin{array}{l}\text { A.M.Goforth, M.D.Smith, H.-C.zur Loye (2003) } \\
\text { J.Chem.Cryst. ,33,303 }\end{array}$} \\
\hline Formula: & \multicolumn{6}{|c|}{$\left(\mathrm{C}_{4} \mathrm{H}_{4} \mathrm{Cu}_{2} \mathrm{I}_{2} \mathrm{~N}_{2}\right) \mathrm{n}$} \\
\hline Compound Name: & \multicolumn{6}{|c|}{ catena-(bis $\left(\mu_{3}\right.$-lodo)-( $\mu_{2}$-pyrazine)-di-copper(i)) } \\
\hline $\begin{array}{l}\text { Space Group: } \\
\text { Space Group No.: }\end{array}$ & $\begin{array}{l}\mathrm{P}-1 \\
2\end{array}$ & $\begin{array}{l}\text { Cell: } \\
(\hat{A}, \circ)\end{array}$ & $\begin{array}{l}a \quad 4.176(0) \\
\alpha 109.61(0)\end{array}$ & $\begin{array}{l}\boldsymbol{b} \quad 7.128(0) \\
\beta 101.77(0)\end{array}$ & & $\begin{array}{l}8.128(0) \\
96.68(0)\end{array}$ \\
\hline R-Factor (\%): & 2.88 & Temp & ure(K): 293 & Density $(g / c n$ & & 3.502 \\
\hline
\end{tabular}

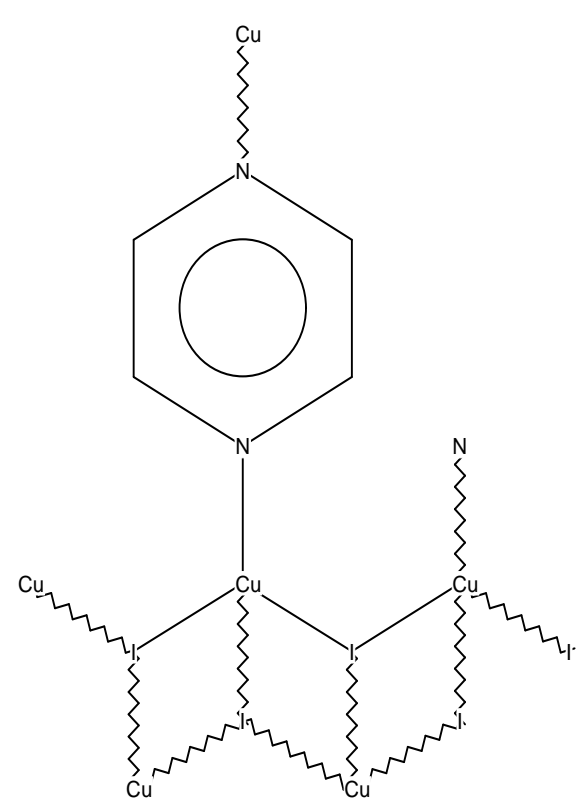

Parameters

Fragment 12053

DEYNIG

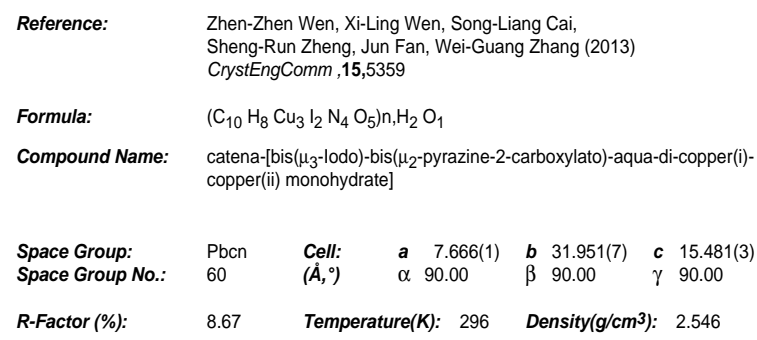

Parameters

Fragment 1

$\begin{array}{ll}\text { Fragment } 1 & \\ \text { DIST1 (D) } & 2.090\end{array}$

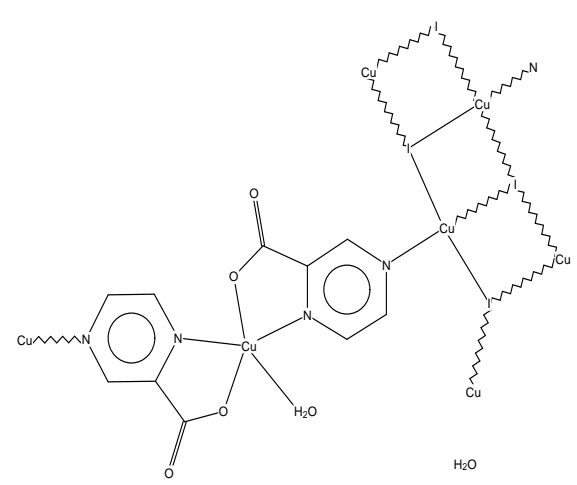




\section{Search: search6 (Sat Mar 07 10:08:52 2020): Hits 5-8}

DIVKUQ

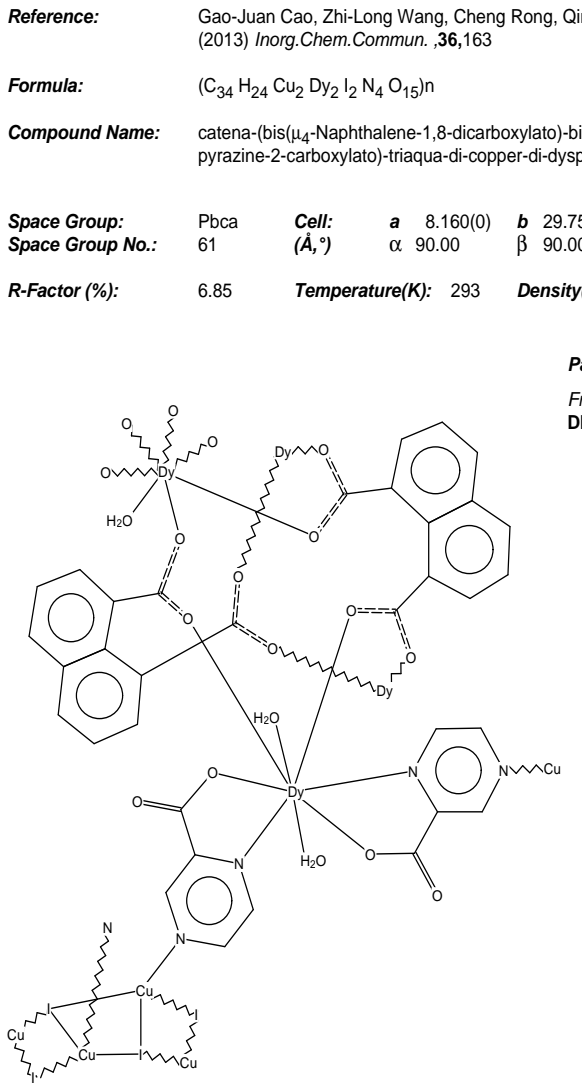

ECEVIS

Reference: J.H.Chong, M.J.MacLachlan (2006) Inorg.Chem. ,45,1442

Formula: $\left(\mathrm{C}_{22} \mathrm{H}_{14} \mathrm{Cu}_{2} \mathrm{I}_{2} \mathrm{~N}_{2}\right) n, n\left(\mathrm{C}_{2} \mathrm{H}_{3} \mathrm{~N}_{1}\right)$

Compound Name: $\quad$ catena-(bis $\left(\mu_{3}\right.$-lodo)-( $\mu_{2}$-5,8-di-azahexacyclo(10.6.6.02,11.04,9.013 copper(i) acetonitrile clathrate)

Synonym: $\quad$ catena-( $\left(\mu_{3}\right.$-lodo)-( $\mu_{2}-2,3-$ pyrazino(b)triptycene)-di-copper(i) acetonitrile clathrate) $\begin{array}{lllllllll}\text { Space Group: } & \text { P21/c } & \text { Cell: } & \boldsymbol{a} & 9.157(1) & \boldsymbol{b} & 31.760(4) & \boldsymbol{c} & 8.246(0) \\ \text { Space Group No.: } & 14 & (\mathbf{A}, \mathcal{\circ}) & \alpha & 90.00 & \beta & 99.38(0) & \gamma & 90.00\end{array}$

R-Factor (\%): $\quad 5.26 \quad$ Temperature(K): $173 \quad$ Density $\left(\mathrm{g} / \mathrm{cm}^{3}\right): 2.045$

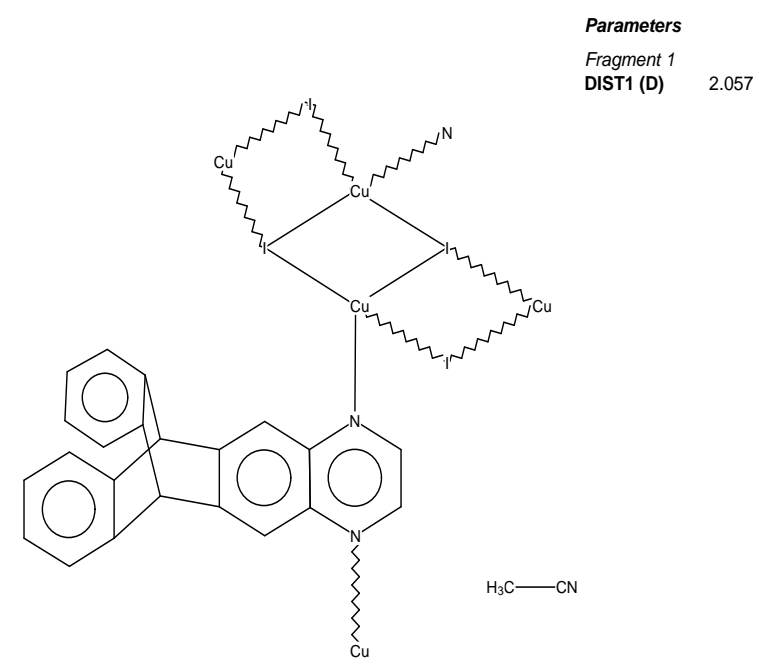

ECEVEO

Reference: J.H.Chong, M.J.MacLachlan (2006) Inorg.Chem. , 45,1442

Formula:

$\left(\mathrm{C}_{28} \mathrm{H}_{17} \mathrm{Cu}_{4} \mathrm{I}_{4} \mathrm{~N}_{7}\right) \mathrm{n}, \mathrm{n}\left(\mathrm{C}_{2} \mathrm{H}_{3} \mathrm{~N}_{1}\right)$

Compound Name:

catena- $\left(\left(\mu_{4}-5,8,16,19,26,29-\right.\right.$ Hexa-azaoctacyclo(10.10.10.02,11.04 $18,20,23(32), 24,26,28,30$-pentadecaene)-( $\mu_{4}$-iodo)-( $\mu_{3}$-iodo)-bis $\left(\mu_{2}\right.$ iodo)-(acetonitrile)-tetra-copper(i) acetonitrile clathrate)

Synonym:

catena-(( $\mu_{4}-$-lodo)-( $\mu_{4}$-tri-2,3-pyrazino(b,e,h)triptycene)-( $\mu_{3}$-iodo)bis $\left(\mu_{2}\right.$-iodo)-(acetonitrile)-tetra-copper(i) acetonitrile clathrate)

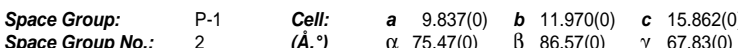
R-Factor (\%):

2.60

Temperature(K): $173 \quad$ Density $\left(\mathrm{g} / \mathrm{cm}^{3}\right): 2.490$

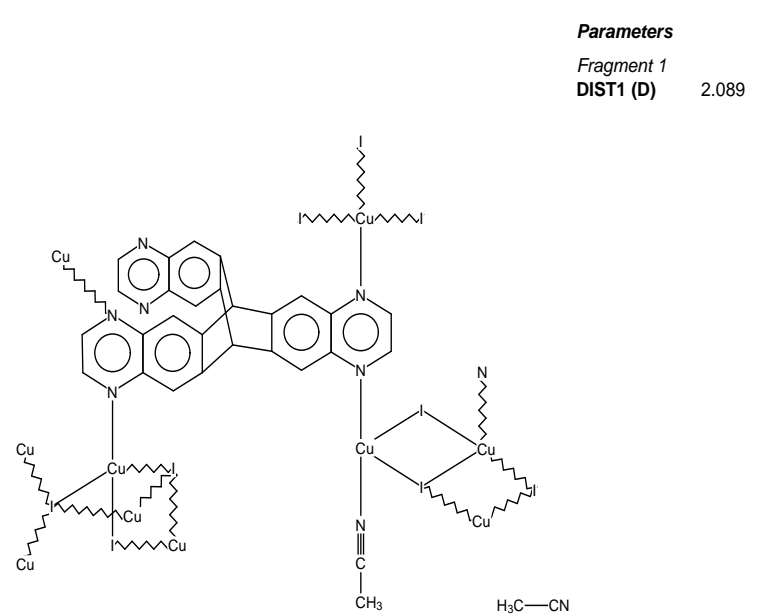

EMELOX

$\begin{array}{ll}\text { Reference: } & \text { C.Nather, I.Jess, N.Lehnert, D.Hinz-Hubner (2003) } \\ & \text { Solid State Sciences ,5,1343 }\end{array}$

Formula: $\quad\left(\mathrm{C}_{12} \mathrm{H}_{16} \mathrm{Cu}_{2} \mathrm{I}_{2} \mathrm{~N}_{4}\right) \mathrm{n}$

Compound Name: $\quad$ catena-( $\left(\mu_{3}\right.$-lodo)-( $\mu_{2}$-2-ethylpyrazine-N, $\left.N^{\prime}\right)-\left(\mu_{2}\right.$-iodo)-(2-ethylpyrazine$\mathrm{N}^{4}$ )-di-copper)

$\begin{array}{lllllllll}\text { Space Group: } & \text { P21/n } & \text { Cell: } & \text { a } 10.434(0) & \boldsymbol{b} & 14.803(0) & \boldsymbol{c} & 11.109(0) \\ \text { Space Group No.: } & 14 & \text { (Aं, }) & \alpha & 90.00 & \beta & 96.71(0) & \gamma & 90.00\end{array}$

R-Factor (\%): $\quad 3.71 \quad$ Temperature(K): $293 \quad$ Density $\left(\mathrm{g} / \mathrm{cm}^{3}\right): 2.328$

\section{Parameters}

Fragment 1

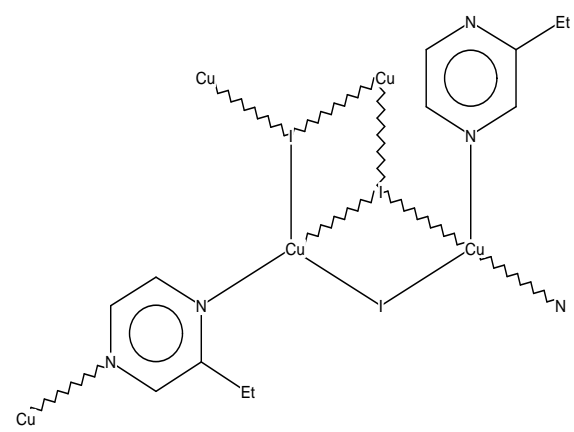




\section{Search: search6 (Sat Mar 07 10:08:52 2020): Hits 9-12}

LIDXUS

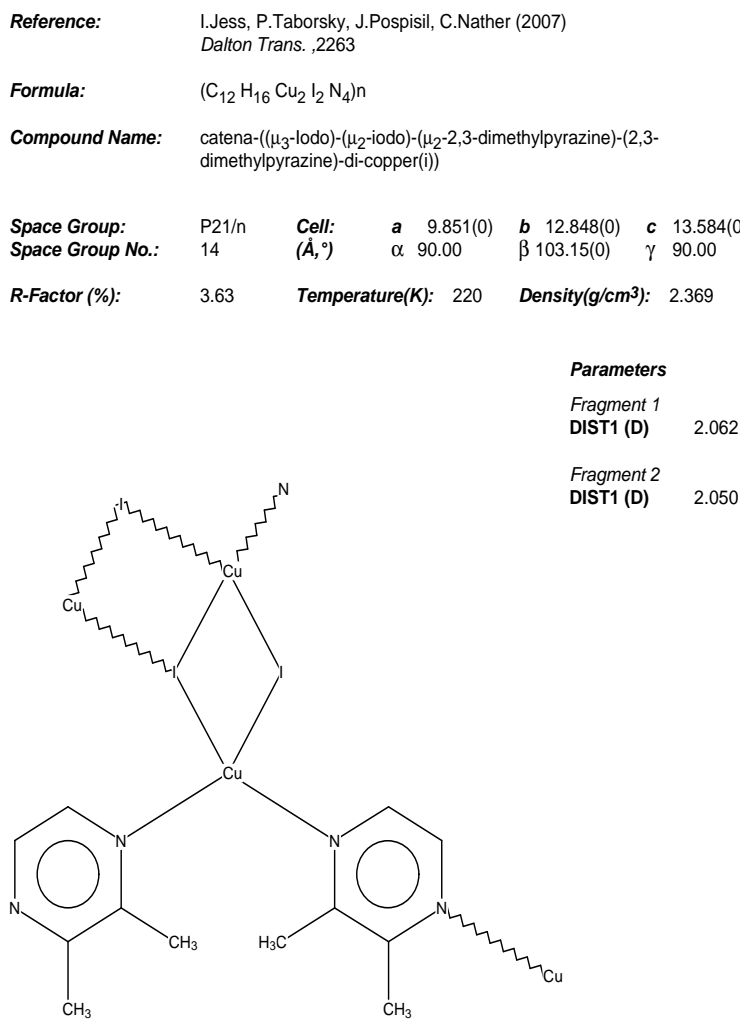

MUHQOV01

\begin{tabular}{|c|c|c|c|c|c|c|}
\hline Reference: & \multicolumn{6}{|c|}{ N.Kitada, T.Ishida (2014) CrystEngComm ,16,8035 } \\
\hline Formula: & \multicolumn{6}{|c|}{$\left(\mathrm{C}_{6} \mathrm{H}_{8} \mathrm{Cu}_{2} \mathrm{I}_{2} \mathrm{~N}_{2}\right) \mathrm{n}$} \\
\hline Compound Name: & \multicolumn{6}{|c|}{ catena-(bis $\left(\mu_{3}\right.$-lodo)-( $\mu_{2}-2,5-$ dimethylpyrazine)-copper(i)) } \\
\hline $\begin{array}{l}\text { Space Group: } \\
\text { Space Group No.: }\end{array}$ & $\begin{array}{l}P-1 \\
2\end{array}$ & $\begin{array}{l}\text { Cell: } \\
\left(\dot{A},{ }^{\circ}\right)\end{array}$ & $\begin{array}{l}a \\
\alpha 110.85(3)\end{array}$ & $\begin{array}{lr}\boldsymbol{b} & 8.220(3) \\
\beta & 102.04(3)\end{array}$ & $\begin{array}{c}c \\
\gamma\end{array}$ & $\begin{array}{l}8.247(3) \\
94.82(3)\end{array}$ \\
\hline R-Factor (\%): & 5.90 & Temp & $r e(K): 293$ & Density $(\mathrm{g} / \mathrm{cm}$ & & 3.097 \\
\hline
\end{tabular}

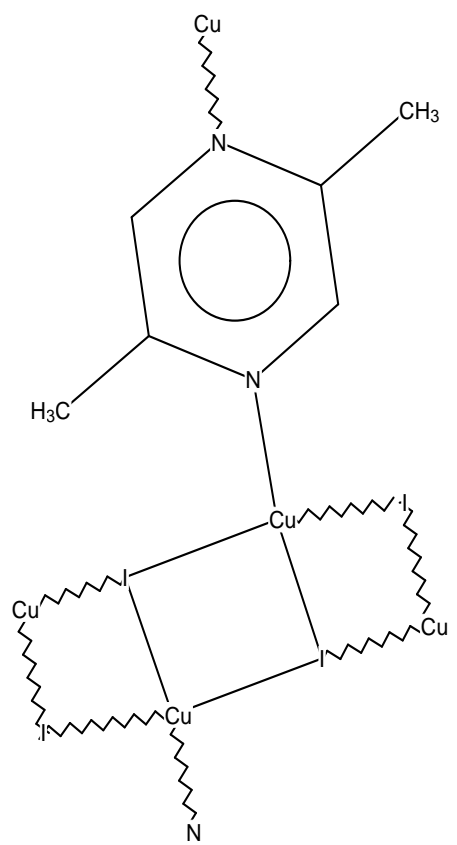

Pameters

Fragment 1 DIST1 (D) 2066
MUHQOV

\begin{tabular}{|c|c|c|c|c|c|c|}
\hline Reference: & \multicolumn{6}{|c|}{$\begin{array}{l}\text { C.Nather, J.Greve, I.Jess (2002) Solid State Sciences ,4, } \\
813\end{array}$} \\
\hline Formula: & \multicolumn{6}{|c|}{$\left(\mathrm{C}_{6} \mathrm{H}_{8} \mathrm{Cu}_{2} \mathrm{I}_{2} \mathrm{~N}_{2}\right) \mathrm{n}$} \\
\hline Compound Name: & \multicolumn{6}{|c|}{ catena-(bis $\left(\mu_{3}\right.$-lodo)-( $\mu_{2}-2,5$-dimethylpyrazine)-di-copper(i)) } \\
\hline $\begin{array}{l}\text { Space Group: } \\
\text { Space Group No.: }\end{array}$ & $\begin{array}{l}P-1 \\
2\end{array}$ & $\begin{array}{l}\text { Cell: } \\
\left(\hat{\boldsymbol{A}},{ }^{\circ}\right)\end{array}$ & $\begin{array}{l}a \quad 4.294(0) \\
\alpha 110.87(1)\end{array}$ & $\begin{array}{l}\boldsymbol{b} \quad 8.181(1) \\
\beta 102.17(1)\end{array}$ & $\begin{array}{c}c \\
\gamma\end{array}$ & $\begin{array}{l}8.215(1) \\
94.54(1)\end{array}$ \\
\hline R-Factor (\%): & 2.31 & Temp & ure(K): 293 & Density $(g / c n$ & & 3.126 \\
\hline
\end{tabular}

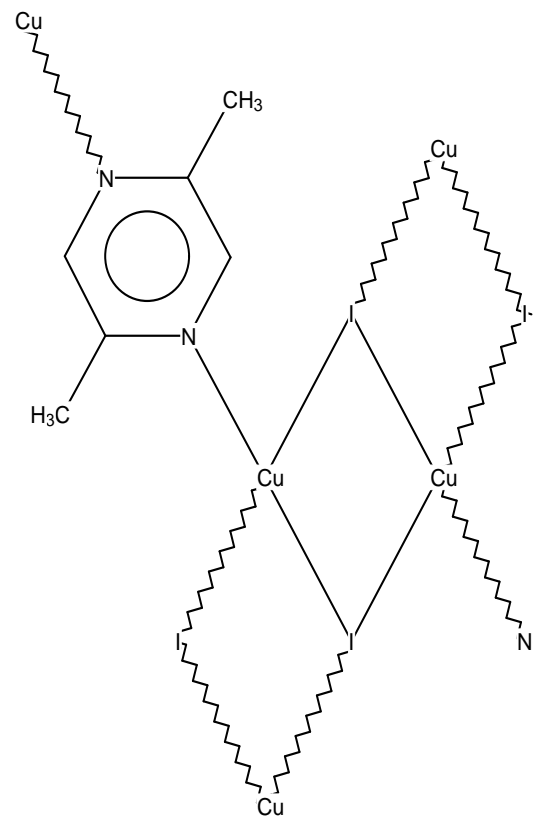

Parameters

2058

\section{RABBUS}

$\begin{array}{ll}\text { Reference: } & \text { J.J.M.Amoore, L.R.Hanton, M.D.Spicer (2003) } \\ \text { Dalton Trans. , 1056 }\end{array}$

Formula: $\quad\left(\mathrm{C}_{12} \mathrm{H}_{14} \mathrm{Cu}_{3} \mathrm{I}_{3} \mathrm{~N}_{4} \mathrm{~S}_{1}\right) \mathrm{n}$

Compound Name: $\quad$ catena-(bis $\left(\mu_{3}-\right.$ lodo)-( $\mu_{3}$-bis(6-methylpyrazin-2-ylmethyl) thioether-N, $\mathrm{N}^{\prime}, \mathrm{N}$ ",S)-($\mu_{2}$-iodo)-tri-copper(i))

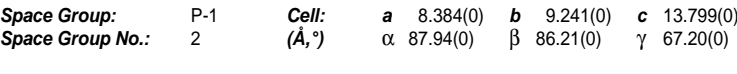

R-Factor (\%): $\quad 2.95 \quad$ Temperature(K): $123 \quad$ Density $\left(\mathrm{g} / \mathrm{cm}^{3}\right): 2.762$

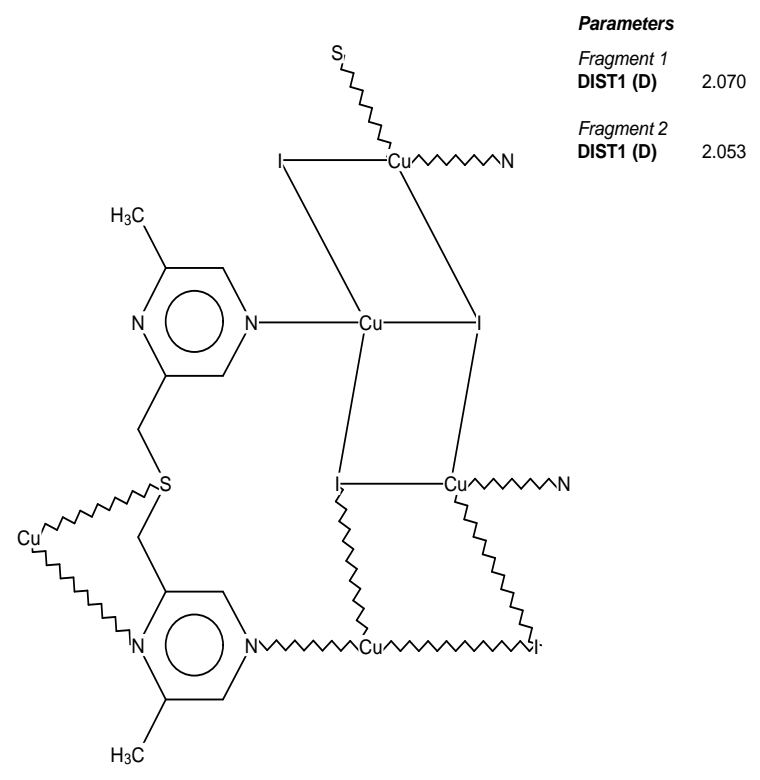




\section{Search: search6 (Sat Mar 07 10:08:52 2020): Hits 13-16}

RUPTED

Reference:

Yu-Jia Ding, Chun-Pei Zhang, Yong-Qing Wang, Xiao-Ming Lin, Ximiao Zhu, Da-Liang Zhang, Xian-Jian Duan, Yue-Peng Cai (2015) CrystEngComm ,17,6693

Formula:

$\left(\mathrm{C}_{10} \mathrm{H}_{8} \mathrm{Cu}_{3} \mathrm{I}_{2} \mathrm{~N}_{4} \mathrm{O}_{5}\right) \mathrm{n}, 0.5 \mathrm{n}\left(\mathrm{H}_{2} \mathrm{O}_{1}\right)$

Compound Name: catena-[bis( $\mu_{3}$-lodo)-bis $\left(\mu_{2}\right.$-pyrazine-2-carboxylato)-aqua-tri-coppe hemihydrate]

$\begin{array}{lllllllll}\text { Space Group: } & \text { Pbcn } & \text { Cell: } & \boldsymbol{a} & 7.668(0) & \boldsymbol{b} & 31.909(3) & \boldsymbol{c} & 15.427(1) \\ \text { Space Group No.: } & 60 & (\mathbf{A}, \boldsymbol{\rho}) & \alpha & 90.00 & \beta & 90.00 & \gamma & 90.00\end{array}$

R-Factor (\%): $\quad 4.22 \quad$ Temperature(K): $296 \quad$ Density $\left(\mathrm{g} / \mathrm{cm}^{3}\right): 2.526$

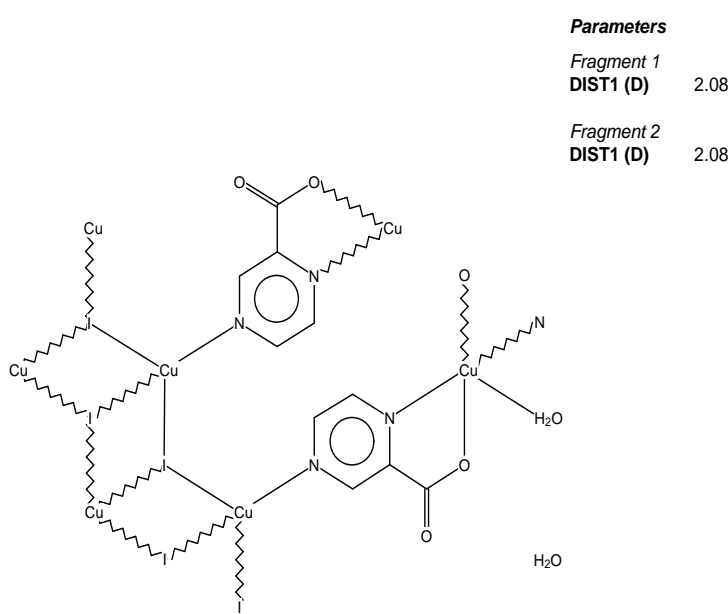

WURYUF

Reference:

T.Hayashi, A.Kobayashi, H.Ohara, M.Yoshida, T.Matsumoto, Ho-Chol Chang, M.Kato (2015) Inorg.Chem. ,54,8905

Formula: $\left(\mathrm{C}_{18} \mathrm{H}_{18} \mathrm{Cu}_{4} \mathrm{I}_{4} \mathrm{~N}_{6}\right) n, n\left(\mathrm{C}_{7} \mathrm{H}_{5} \mathrm{~N}_{1}\right)$

Compound Name: catena-( $\left(\mu_{4}-2,2^{\prime}, 2^{\prime \prime}\right.$-Cyclohexane-1,3,5-triyltripyrazine)-tetrakis $\left(\mu_{3}\right.$-iodo)tetra-copper benzonitrile solvate) $\begin{array}{lllllllll}\text { Space Group: } & \text { P21/c } & \text { Cell: } & \boldsymbol{a} & 14.765(5) & \boldsymbol{b} & 17.932(6) & \boldsymbol{c} & 16.285(5) \\ \text { Space Group No.: } & 14 & \left(\dot{\boldsymbol{A}},{ }^{\circ}\right) & \alpha & 90.00 & \beta & 105.68(0) & \gamma & 90.00\end{array}$ R-Factor (\%): $\quad 6.26 \quad$ Temperature(K): $100 \quad$ Density $\left(\mathrm{g} / \mathrm{cm}^{3}\right): 1.893$

Parameters

Fragment 1

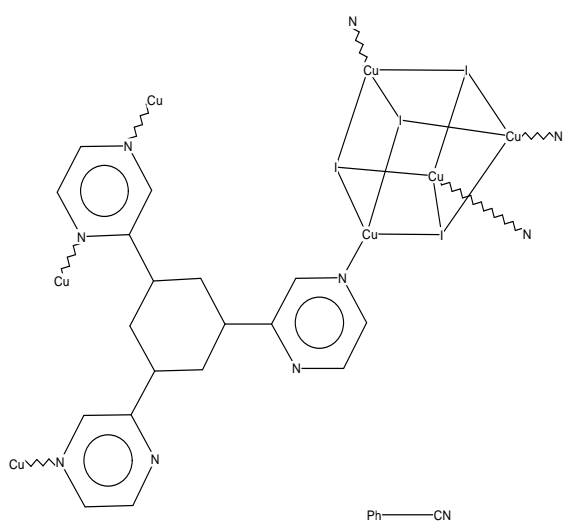

VEVGUY

Reference: $\quad$ P.M.Graham, R.D.Pike, M.Sabat, R.D.Bailey, W. T. Pennington (2000) Inorg Chem. 39,5121

Formula:

$\left(\mathrm{C}_{8} \mathrm{H}_{6} \mathrm{Cu}_{2} \mathrm{I}_{2} \mathrm{~N}_{2}\right) \mathrm{n}$

Compound Name: $\quad$ catena-(bis $\left(\mu_{3}\right.$-iodo)-( $\mu_{2}$-quinoxaline-N,N')-di-copper(i))

Space Group: $\quad$ P21/c $\quad$ Cell: $\quad$ a $4.372(0)$ b 17.718(2) $\quad$ c $14.748(0)$ $\begin{array}{lllllll}\text { Space Group No.: } \quad 14 & \left(\dot{A},{ }^{\circ}\right) & \alpha & 90.00 & \beta 110.16(0) & \gamma & 90.00\end{array}$

R-Factor (\%): $\quad 6.18 \quad$ Temperature(K): $295 \quad$ Density(g/cm3): 3.165

\section{Parameters}

Fragment 12046

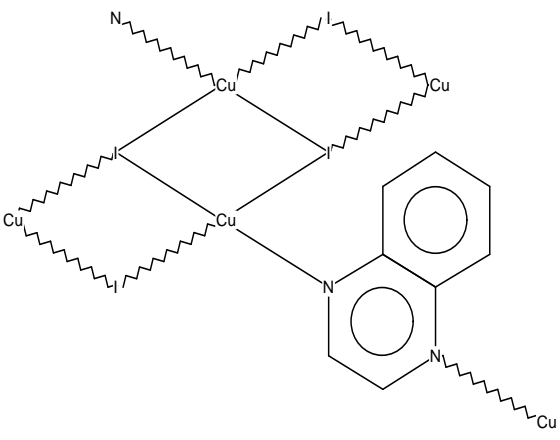

ZEBNUP

Reference: $\quad$ M.A.S.Goher, T.C.W.Mak (1995) Polyhedron ,14,2587

Formula:

$\left(\mathrm{C}_{10} \mathrm{H}_{8} \mathrm{Cu}_{2} \mathrm{I}_{2} \mathrm{~N}_{4} \mathrm{O}_{4}\right) \mathrm{n}, 3 \mathrm{n}\left(\mathrm{H}_{2} \mathrm{O}_{1}\right)$

Compound Name: $\quad$ catena(bis(( $\mu_{3}$-lodo)-(pyridine-2-carboxylate)-copper(ii)) trihydrate)

Space Group: $\quad$ P-1 $\quad$ Cell: $\quad \begin{array}{lllllll}\text { a } & 6.813(1) & \boldsymbol{b} & 8.273(1) & \boldsymbol{c} & 16.719(2)\end{array}$

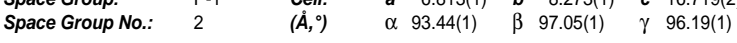

R-Factor (\%): $\quad 3.47 \quad$ Temperature(K): $295 \quad$ Density(g/cm 3 ): 2.447

Parameters

Fragment 1

$\begin{array}{ll}\text { Fragment } 2 & \\ \text { DIST1 (D) } & 2.053\end{array}$

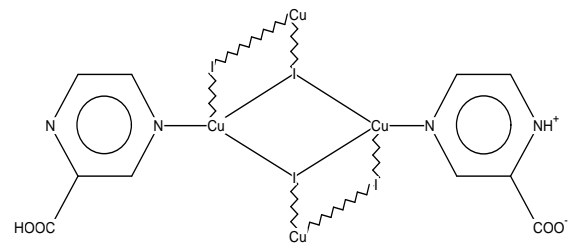

$\mathrm{H}_{2} \mathrm{O}$ 